\title{
Asteroseismology of pre-main sequence stars
}

\author{
K. Zwintz, ${ }^{1}$ D. B. Guenther, ${ }^{2}$ and T. Kallinger ${ }^{1}$ \\ ${ }^{1}$ Institut für Astronomie, Universität Wien, Türkenschanzstrasse 17, 1180 Vienna, Austria \\ 2 Department of Astronomy and Physics, St. Mary's University, Halifax, NS B3H 3C3, Canada
}

\begin{abstract}
Intermediate-mass pre-main sequence (PMS) stars cross the instability region on their way to the main sequence and can become pulsationally unstable. The $\delta$ Scuti-like PMS pulsators populate the same area in the Hertzsprung-Russell (HR) diagram as the classical $\delta$ Scuti stars. But the interiors of PMS stars differ from those of their more evolved (post-)main sequence counterparts resulting in different pulsation frequency spectra. Asteroseismology of PMS p-mode pulsators has only recently become possible. The latest investigation focused on the analysis of MOST space photometry of the Herbig Ae star HD 142666 finding 12 pulsational frequencies lying on top of larger irregular variability caused by a circumstellar disk.
\end{abstract}

Individual Objects: HD 142666

\section{Introduction}

Young stars mainly gain their energy from gravitational contraction before they arrive on the zero-age main sequence (ZAMS). Their interiors show different density profiles compared to their (post-)main sequence counterparts (Marconi \& Palla 1998) and lack regions of already processed nuclear material. Such pre-main sequence (PMS) objects cross the instability region in the HR diagram while evolving to the ZAMS and can become pulsationally unstable. As the stellar density profiles in PMS stars are different than in (post-)main sequence stars of same effective temperature, luminosity and mass, the pulsational frequency spacings also differ for the two evolutionary stages (Suran et al. 2001). Hence, with a dense enough pulsational frequency spectrum, asteroseismology can be used to investigate the evolutionary stage of a field star.

\section{The PMS Instability Strip}

For the 36 known PMS pulsators (i.e., 18 Herbig Ae field stars and 18 members of young open clusters), a comparison to the classical $\delta$ Scuti stars was conducted (Zwintz 2008). While a wealth of astrophysical parameters are available for the classical $\delta$ Scuti objects, the only common parameters of the 36 pulsating PMS stars are Johnson $V$ and $(B-V)$ or their corresponding dereddened values of $M_{V}$ and $(B-V)_{0}$. The comparison of these pulsators in the observed HR diagram (in the $M_{V}$ vs. $(B-V)_{0}$ plane) showed that $\delta$ Scuti stars of both evolutionary stages occupy the same instability region (Zwintz 2008). 


\section{HD 142666}

Only one PMS pulsator is located redwards of the red boundary of the classical $\delta$ Scuti instability strip: HD 142666. This Herbig Ae star (spectral type A8 Ve and $V=8.81 \mathrm{mag}$ ) is known to have a dense circumstellar dust disk seen nearly edge-on (e.g., Meeus et al. 1998) that causes irregular brightness variations of up to a magnitude peak-to-peak which are accompanied by colour changes. When the dust clouds are in the observer's line-ofsight, they obscure the star and cause the reddening. As the star is never seen free from its circumstellar dust, its $(B-V)_{0}$ can only be determined with large relative errors which explains HD 142666's "extreme" location in the $M_{V}$ vs. $(B-V)_{0}$ plane.

Kurtz \& Müller (2001) discovered a single pulsation frequency of $21.43 \mathrm{~d}^{-1}$ (i.e., period of 1.12 hours) for HD 142666. In 2006 and 2007 the MOST (Microvariability and Oscillations of Stars) satellite (Walker et al. 2003) observed HD 142666 for 11.5 days and 39 days, respectively. 12 pulsational frequencies were identified in the data sets of both years and were submitted to a detailed asteroseismic analysis (for more details see Zwintz et al. 2008). Good model fits were obtained to the frequencies for $I=0,1$ and 2 p modes, but all models lie well outside the observationally derived HR diagram uncertainty box of HD 142666. The reason for this could be that the published value for HD 142666's luminosity is overestimated and/or that additional physics would have to be included in the models to find a good fit to the observed frequencies and the star's position in the HR-diagram (Zwintz et al. 2008).

Acknowledgments. K.Z. and T.K. acknowledge support from the Austrian Science Funds (FWF, KZ: project T335-N16, TK: project P17580). The Natural Sciences and Engineering Research Council of Canada supports the research of D.B.G.

\section{References}

Kurtz, D. W., \& Müller, M. 2001, MNRAS, 325, 1341

Marconi, M., \& Palla, F. 1998, AJ, 507, L141

Meeus, G., Waelkens, C., \& Malfait, K. 1998, A\&A, 329, 131

Suran, M., Goupil, M., Baglin, A., et al. 2001, A\&A, 372, 233

Walker, G., Matthews, J. M., Kuschnig, R., et al. 2003, PASP, 115, 1023

Zwintz, K. 2008, ApJ, 673, 1088

Zwintz, K., Kallinger, T., Guenther, D. B., et al. 2009, A\&A, 494, 1031

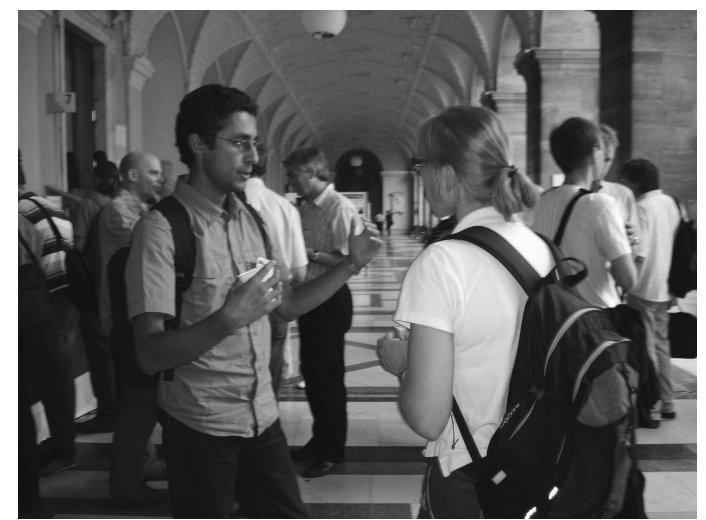

